Online Supporting Information for

\title{
Work Function Engineering of 2D Materials: The Role of Polar Edge Reconstructions
}

\author{
Guoxiang $\mathrm{Hu}^{*, \dagger,}$, Victor Fung ${ }^{\dagger}$, Jingsong Huang ${ }^{\dagger}$, and P. Ganesh ${ }^{*, \dagger}$ \\ guoxiang.hu@qc.cuny.edu, ganeshp@ornl.gov \\ $\dagger$ Center for Nanophase Materials Sciences, Oak Ridge National Laboratory, Oak Ridge, \\ Tennessee 37831, United States \\ $\$$ Department of Chemistry and Biochemistry, Queens College of the City University of New \\ York, Queens, New York 11367, United States
}




\section{Computational methods}

Spin-polarized density functional theory (DFT) calculations were performed using the Vienna ab initio simulation package (VASP). ${ }^{1-2}$ Electron exchange-correlation was represented by the functional of Perdew, Burke, and Ernzerhof (PBE) of generalized gradient approximation (GGA). ${ }^{3}$ The ion-electron interaction was described with the projector-augmented wave (PAW) method. ${ }^{4}$ The plane-wave cutoff is set to $400 \mathrm{eV}$, and a conjugate gradient method is applied to relax the geometry until interatomic forces are less than $0.025 \mathrm{eV} / \AA$. The Brillouin zone was sampled using a $3 \times 1 \times 1$ Monkhorst-Pack k-point meshes for the $4 \times 1 \times 1$ supercells. Vacuum spaces in y and $\mathrm{z}$ directions are larger than $20 \AA$, and dipole corrections along y were used. To test the convergence of the thickness of vacuum along y, we doubled the size of the supercell shown in Figure 1 along y. This increases the vacuum space along y from $24 \AA$ to $64 \AA$. As shown in Figure S4, the work functions at the two edges change only by about $0.1 \mathrm{eV}$ as the vacuum thickness increases over 2 times (the Mo side increases by $0.11 \mathrm{eV}$ and the $\mathrm{S}$ side decreases by $0.08 \mathrm{eV}$ ). This demonstrates that the vacuum used in our calculations is thick enough to avoid interactions between the two edges. The plane-averaged electrostatic potential energy and charge density considered only the regions determined by the ribbon width and thickness. Partial atomic charges were obtained using Bader charge analysis as implemented by Henkelman and coworkers. ${ }^{5}$ Isosurfaces of charge density difference were visualized using the VESTA program. ${ }^{6}$

The formation energy per atom was calculated by

$$
E_{f} / A=\frac{E_{t o t}\left(M o_{x} S_{y}\right)-x \mu_{M o}-y \mu_{S}}{x+y}
$$

where $E_{\text {tot }}\left(M o_{x} S_{y}\right)$ is the total energy of the Mox $S_{\mathrm{y}}$ nanoribbon, and $\mu$ Mo and $\mu$ s are the chemical potentials of Mo and S. The chemical potentials are $\mu$ Mo in body-centered cubic (bcc) Mo and $\mu \mathrm{s}$ in orthorhombic alpha-S, which were obtained from the computed energies per atom at $\mathrm{T}=0 \mathrm{~K}$ 
by neglecting the entropic terms for the condensed states (i.e. $\mu \mathrm{s}($ bulk $) \approx \mathrm{Es}(\mathrm{bulk})=-4.2 \mathrm{eV}$, and $\left.\mu_{\mathrm{Mo}}(\mathrm{bulk}) \approx \mathrm{E}_{\mathrm{Mo}}(\mathrm{bulk})=-11.5 \mathrm{eV}\right)$.

The many-body tensor representation features were calculated using the Dscribe python package. ${ }^{7}$ A principal component analysis (PCA) was first performed on the many-body tensor representation features to reduce noise, selecting the first 30 components capturing $99 \%$ of the variance, prior to the t-distributed Stochastic Neighbor Embedding (t-SNE) analysis. The t-SNE analysis was done using the corresponding sci-kit learn python package. ${ }^{8}$ The primary parameter determining the distribution in t-SNE, the perplexity, was set to be 20 for both representations. 
(a)

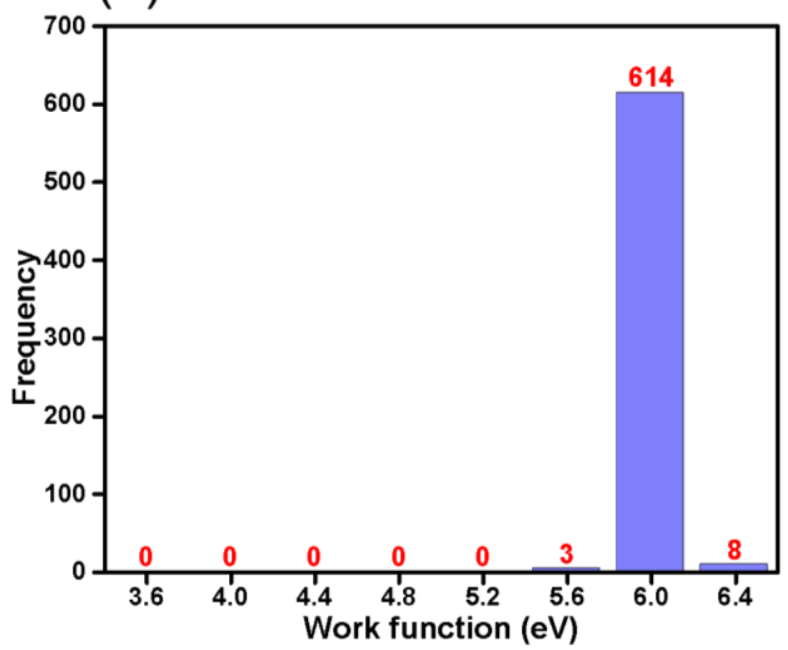

(b)

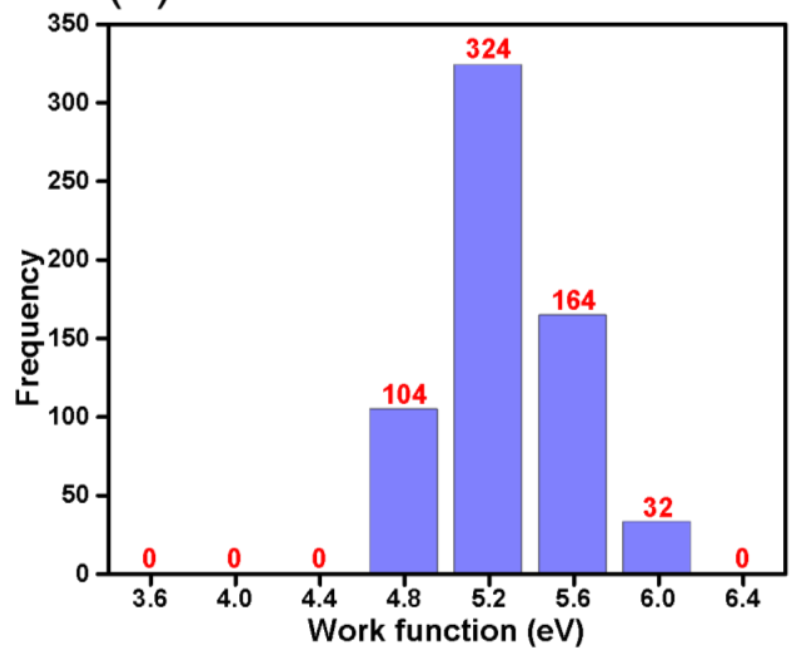

Figure S1. Histograms of the work function (a) at the S side along the width of the ribbon and (b) along the normal direction for the 625 nanoribbons with reconstructions only at the Mo side.

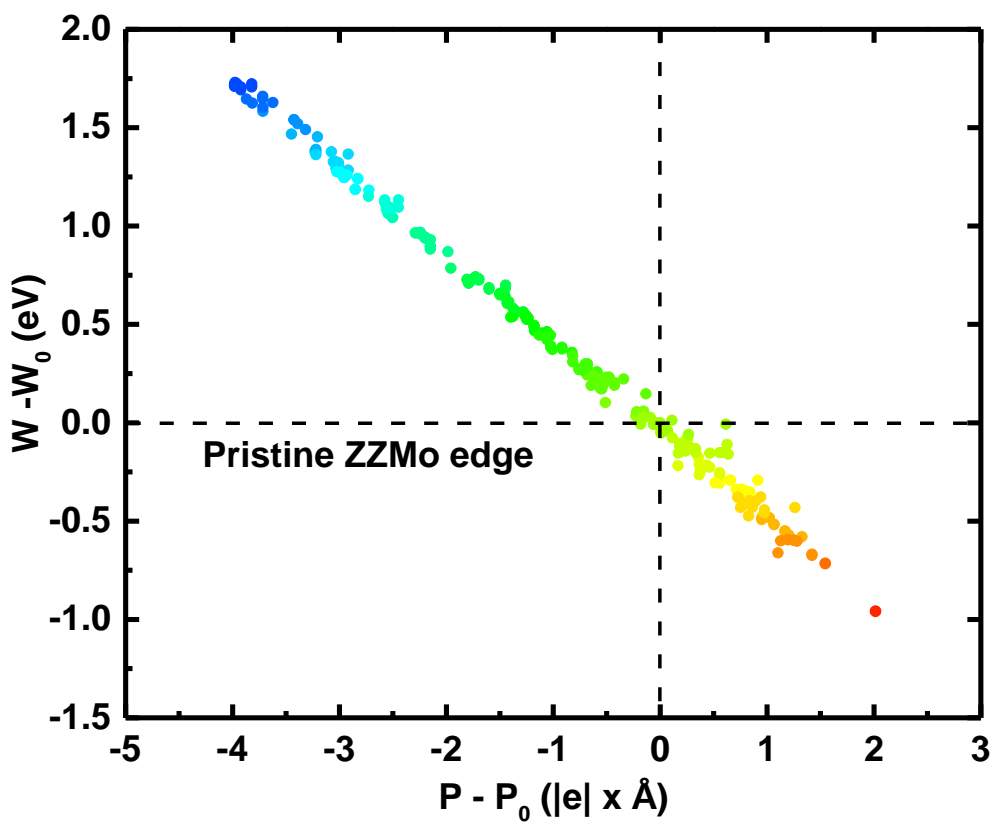

Figure S2. Linear correlations between the change in the work function $\left(\mathrm{W}-\mathrm{W}_{0}\right)$ and the change in the edge dipole moment ( $\left.\mathrm{P}-\mathrm{P}_{0}\right)$ at the Mo side for the 267 stable edges. Different colors of data points are assigned based on their work function. 


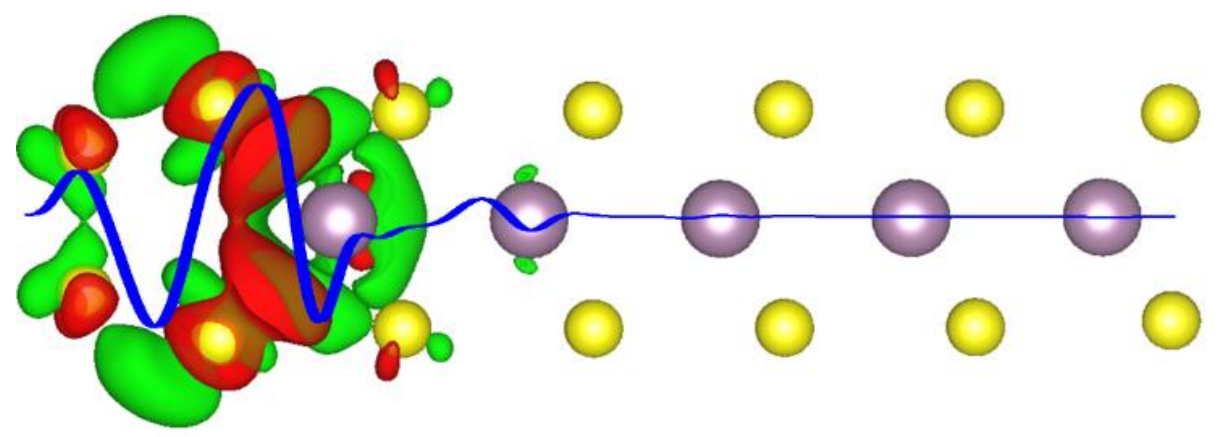

Figure S3. Edge reconstruction induced change in the charge density for the nanoribbon with the S-rich ZZMo-S4 edge. The charge density difference $\Delta \rho$ was defined as $\Delta \rho=\rho$ (total) $-\rho$ (pristine)- $\rho$ (edge), where $\rho$ (total) is total charge density of the ribbon, $\rho$ (pristine) is the charge density of the pristine region in the ribbon, and $\rho($ edge) is charge density of the freestanding reconstructed edge. The red color indicates charge accumulation, whereas the green color indicates charge depletion. The isosurface value is $0.0025 \mathrm{eV}^{-3}$. The plane-averaged charge density difference along the width of the ribbon is also shown with the blue curve. S, yellow; Mo, grey.

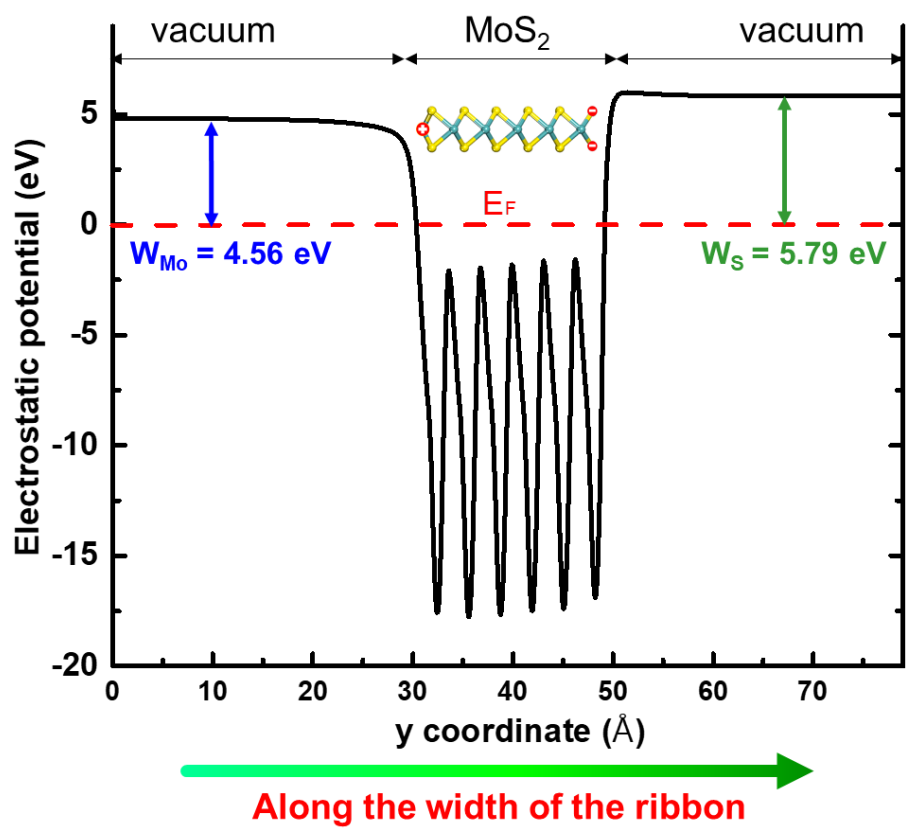

Figure S4. Plane-averaged electrostatic potential energy of a pristine $\mathrm{MoS}_{2}$ nanoribbon along y coordinates for a vacuum thickness of $64 \AA$. 


\section{References}

(1) Kresse, G.; Furthmüller, J. Efficient Iterative Schemes for Ab Initio Total-Energy Calculations Using a Plane-Wave Basis Set. Phys. Rev. B 1996, 54, 11169-11186.

(2) Kresse, G.; Furthmüller, J. Efficiency of Ab-Initio Total Energy Calculations for Metals and Semiconductors Using a Plane-Wave Basis Set. Comput. Mater. Sci. 1996, 6, 15-50.

(3) Perdew, J. P.; Burke, K.; Ernzerhof, M. Generalized Gradient Approximation Made Simple. Phys. Rev. Lett. 1996, 77, 3865-3868.

(4) Blöchl, P. E. Projector Augmented-Wave Method. Phys. Rev. B 1994, 50, 17953-17979.

(5) Tang, W.; Sanville, E.; Henkelman, G. A Grid-Based Bader Analysis Algorithm without Lattice Bias. J. Phys.: Condens. Matter 2009, 21, 084204.

(6) Momma, K.; Izumi, F. VESTA 3 for Three-Dimensional Visualization of Crystal, Volumetric and Morphology Data. J. Appl. Crystallogr. 2011, 44, 1272-1276.

(7) Himanen, L.; Jäger, M. O.; Morooka, E. V.; Canova, F. F.; Ranawat, Y. S.; Gao, D. Z.; Rinke, P.; Foster, A. S. Dscribe: Library of Descriptors for Machine Learning in Materials Science. Comput. Phys. Commun. 2020, 247, 106949.

(8) Pedregosa, F.; Varoquaux, G.; Gramfort, A.; Michel, V.; Thirion, B.; Grisel, O.; Blondel, M.; Prettenhofer, P.; Weiss, R.; Dubourg, V.; et al. Scikit-Learn: Machine Learning in Python. J. Mach. Learn. Res. 2011, 12, 2825-2830. 